nellen Staatlichkeit nicht von einem Staatenbund gesprochen werden kann“ (S. 197) - und zum Bestandsschutz der (Bundes-)Staatlichkeit in Art. 79 Abs. 3 GG nicht überschreiten dürfe: „Nur ein bürgernaher und demokratisch legitimierter europäischer vierstufiger Bundesstaat mit einem Staatsaufbau von unten nach oben, beginnend mit der Kommunalität, gefolgt von Ländern und Regionen, den Mitgliedstaaten und schließlich einer lediglich funktional-staatlichen Union kann verhindern, dass aus dem Konzept einer Friedensordnung eine Machtordnung wird." (S. 378) Alle Staaten entstanden durch Kriege; erst die EU ist eine friedliche Einigung, die im Nationalstaat vernachlässigte Verfassungsnormen wie Gleichberechtigung der Frau, Diskriminierungsverbot, Umweltschutz realisiert und deren Euro Schuldenfallen verhindert. „Die europäische Einheit, die sich auf Papier - auch demjenigen einer Verfassung oder eine Landkarte - nicht herstellen, im Kopf nicht begründen läßt, schleicht sich gewissermaßen als europäische Größe ein und wird in dieser Form eine Tatsache und als solche unwiderleglich. "1 All das übersieht diese überlange Arbeit (über die Hälfte Fußnoten) mit überholten Maßstäben, die trotz allem ein gutes Kompendium verfassungsrechtlicher Literatur ist.

Erich Röper

1 AdolfMuschg, Was ist europäisch? Reden für einen gastlichen Erdteil, Bonn 2005, S. 28.

\title{
Wechselspiel aus Politik und Recht
}

Massing, Otwin: Politik als Recht - Recht als Politik. Studien zu einer Theorie der Verfassungsgerichtsbarkeit, Nomos Verlagsgesellschaft, Baden-Baden 2005, 276 Seiten, € 44,-.

Francis Fukuyamas These vom Ende der Geschichte nach dem Ende der Ost-West-Konfrontation bleibt fragwürdig: Die Geschichte endete nicht, sie bog nur anders ab. Neu entstand zudem die Frage, inwiefern sich Deutungs- und Kritikmuster, deren Legitimität und Brisanz vorwiegend auf dem bisherigen politischen Antagonismus beruhten, auf die Situation nach 1989/1990 anwenden lassen. Dies interessiert vorwiegend dann, wenn gesellschaftliche Prozesse vor dem Hintergrund mindestens zweier methodischer Systeme analysiert werden, von denen eines „Politik“ heißt. Denn sowohl die Allokation sich auf öffentliche Legitimation stützender Macht im internationalen Gefüge als auch ihre ideologischen Begründungszusammenhänge änderten sich in Folge der tektonischen Verschiebungen nach 1989/1990. Im Rahmen einer interdisziplinären Analyse eignet sich das Recht hervorragend als Kontrast zur Politik, weil es den Nimbus der Entscheidungsklarheit allein aus dem Wortlaut der Rechtsquellen trägt. Insofern diese positivistisch allenfalls als geronnene Politik interpretiert werden, könnte man zu der Auffassung gelangen, das Recht habe die Politik gleichsam hinter sich gelassen. Ist dies so? Oder inwiefern bilden sich wechselseitige Abhängigkeiten zwischen den - juristischen beziehungsweise politologischen - Deutungszugängen und deren historischen Entwicklungen? „Politik als Recht - Recht als Politik“ heißt das Sammelwerk von Otwin Massing, das dem an der eigenen Antwort auf diese 
Fragen interessierten Leser empfohlen werden kann. Seine zwölf Kapitel bestehen aus bereits anderweitig, etwa in dieser Zeitschrift, publizierten Beiträgen aus den Jahren 1967 bis 2000 - ein für die Bundesrepublik polithistorisch gewaltiger Zeitraum. Die Schriften Massings werden dabei zur Spektralanalyse materialistischer Kritik an dem Wechselspiel zwischen Recht und Politik in der Bundesrepublik. Den biografischen Hintergrund erläutert der Autor in seinem Vorwort: Er hatte ein Habilitationsstipendium für eine Arbeit erhalten, die „Das Entscheidungsideal des Bundesverfassungsgerichts“ heißen sollte. Zwischenzeitlich durch eine Berufung zum Titularprofessor aufgestiegen, nahm er von dem Vorhaben Abstand. Der kritische Zugang insbesondere zu Struktur und Judikatur des Bundesverfassungsgerichts (BVerfG) als demjenigen Gericht, an dem sich Politik und Recht am nächsten kommen dürften, begleitete den Autor gleichwohl. Unter anderem daher wurde er zur Federführung einer Enquête-Kommission der Deutschen Vereinigung für Politische Wissenschaften zu universitären Mitbestimmungsmöglichkeiten berufen, woraus der Beitrag „,Gruppenuniversität' und ,forschendes Lernen”“ erwuchs.

Sprachlich wie inhaltlich ist den Beiträgen eine gegenüber dem bundesrepublikanischen (Verfassungs-)Rechtssystem grundskeptische, teilweise fundamentaloppositionelle Haltung immanent, die bedauerlicherweise gelegentlich wertvolle Erkenntnisse überdeckt. Dies sei an zwei Beispielen erläutert. Im Kapitel „Von der Volkszählungsboykottbewegung zur Verrechtlichung, oder: Öffentlichkeit, Herrschaftsrationalisierung und Verfahren" setzt sich der Verfasser mit dem Öffentlichkeitsprinzip als politisch-normativer Maxime am Beispiel der geplanten Volkszählung von 1983 auseinander. Er entwickelt zunächst einen partizipativen Öffentlichkeitsbegriff, behauptet sodann die Okkupation der Macht durch das Bürgertum im Wege eines politischen Enteignungsvorgangs, um danach im Hinblick auf die Öffentlichkeit festzustellen, sie sei in der „spätkapitalistischen Entwicklungsphase“ ein „neofeudales Kondominium“ unter Einschluss „neokorporatistischer Einflussmächte“ (S. 169). Diese Analyse erscheint nicht nur aus heutiger Sicht sprachlich unmodern. Sie lenkt insbesondere von dem bedenkenswerten Ansatz des Verfassers ab, dass sich Öffentlichkeit in einer pluralistischen Gesellschaft im Wesentlichen medial vollzieht, größtenteils erst konstituiert, und wegen der - politischen, rechtlichen, aber eben auch ökonomischen - Einflüsse auf die Medien (die) Öffentlichkeit von den jeweiligen Funktionseliten stärker geprägt wird, als dies der Anspruch allgemeiner Partizipation nahe legt. Dieses Problem auf den ökonomischen Aspekt zu reduzieren, steht in der marxistischen Tradition, gesellschaftliche Differenzen in Haupt- und Nebenwidersprüche einzuteilen und als Hauptwiderspruch stets die Ökonomie zu identifizieren. But it isn't always the economy.

Im Weiteren ruft der Beitrag unter den diskutierten Widerstandsformen gegen die Volkszählung auch den Rechtsweg vor das BVerfG auf. Dieses verhinderte damals die Volkszählung, weil es das - anhand dieses Falls aus den Grundrechten entwickelte - Recht auf informationelle Selbstbestimmung verletzt sah. Der Autor interpretiert das Judikat als Ausdruck einer „Strategie prozeduraler Herrschaftsrationalisierung“ (S. 183) und konzediert, die Domestizierung des politischen Widerstandes sei und habe System. Tatsächlich bot das Verfahren eine beispielhafte Anschauung dafür, wie eine Widerstandsbewegung ihre Kritik an der befürchteten Überwachung vom BVerfG bestätigt sah, sich formal kriminalisierter Widerstandsformen enthalten konnte und somit Rechtsfrieden hergestellt wurde. Der Autor würdigt dieses Ergebnis jedoch nicht positiv, sondern zitiert die fundamentalen Kritiker der Entscheidung so ausgiebig, dass jedenfalls der Eindruck entsteht, auch er bedauere, dass aufgrund des Erfolgs in Karlsruhe „dem eigentlichen Widerstand die Luft“ 
(S. 182) abgedreht worden sei. Durch diese buchstäblich ein-seitige Sichtweise tritt die beachtenswerte Kritik allzu sehr in den Hintergrund: „Demokratie muss hinfort durchs Nadelöhr der Justiz." (S.185) In der Tat. Diese Prä-Position, die die justizförmige Konfliktbewältigung gegenüber dem demokratischen Diskurs gewinnt, indem fundamentale Streitfragen durch Gerichte beantwortet werden, bedurfte und bedarf der vertieft analytischen und kritischen Würdigung. Auch der eher skizzenhafte Beitrag „Zur Rolle und Funktion der wissenschaftlichen Mitarbeiter im Entscheidungsprozeß des Bundesverfassungsgerichts - oder: eine juridische ,black box' als Forschungsgegenstand?" belegt das grundsätzliche Dilemma der Publikation: Einerseits macht der Verfasser treffsicher interessante Sujets ausfindig und konfrontiert sie mit brisanter Struktur- und Prozesskritik - so etwa, wenn er die These eines institutionell honorierten affirmativen Konformismus' der wissenschaftlichen Mitarbeiter beim BVerfG entwickelt. Andererseits bleibt er dem Primat der Ökonomie verhaftet: Seine Deutung des BVerfG als „Karlsruher Prozessbörse“ (S. 187) beispielsweise, an der die Nachfrage nach Entscheidungen befriedigt werden muss, wird nicht nur der Würde der Richter nicht gerecht, die sich kaum mit einer Rolle als Makler und Verkäufer in einer Person identifizieren dürften. Sie bietet darüber hinaus keinen methodischen Mehrwert.

Einem Leser, der sich auf die dem Charakter als Sammelband von Beiträgen aus 33 Jahren inhärente Zeitreise ebenso einlässt wie auf deren teilweise drastische Sprache, bietet die Lektüre von „Politik als Recht - Recht als Politik“ insbesondere zweierlei: erstens eine Fülle von Kritiken an Struktur und Folgen der Rechtsprechung des BVerfG (und, im letzten Beitrag, des Europäischen Gerichtshofes), die im verfassungsrechtlichen Binnendiskurs selten diskutiert werden und schon daher zum Weiterdenken und gegebenenfalls -forschen einladen. Und zweitens die Möglichkeit zu eigenen Antworten auf die Frage nach der Wechselbezüglichkeit zwischen analytischen Methoden und ihren historischen Hintergründen.

Sebastian Lovens

\section{Schlüsselwörter in der Politik}

Shrouf, A. Naser: Sprachwandel als Ausdruck politischen Wandels. Am Beispiel des Wortschatzes in Bundestagsdebatten 1949-1998, Peter Lang Verlag, Frankfurt am Main u.a. 2006, 349 Seiten, $€ 56,50$.

Politik und Sprache stehen in einem engen Verhältnis, und beide wandeln sich. Die Frage ist, wie ihre Veränderungen sich gegenseitig bedingen. A. Naser Shrouf bezieht im Titel seiner sprachwissenschaftlichen Dissertation eine klare Position. Er sieht den Wandel von Sprache „als Ausdruck politischen Wandels“, letzterer ziehe Veränderungen ersterer nach sich. Politikwandel versteht der Autor als Regierungswechsel, eng bezogen auf den Wechsel des Hauptkoalitionspartners zwischen Union und SPD. Hauptsächlich interessiert ihn, „wie und inwiefern sich der Politikwechsel in der Sprache der Politik in Deutschland niederschlägt" (S. 3) und wie das Sprachverhalten der jeweiligen Partei realisiert wird (in Regierungs- wie in Oppositionszeiten). 